\title{
INFLUÊNCIA DA ACURÁCIA ALTIMÉTRICA DO MODELO DIGITAL DO TERRENO NA ELABORAÇÃO DO ÍNDICE DE CONCENTRAÇÃO DE RUGOSIDADE GLOBAL (ICR - GLOBAL)
}

\author{
Otacílio Lopes de Souza Paz ${ }^{(a)}$, Tony Vinícius Moreira Sampaio ${ }^{(b)}$
}

(a) Departamento de Geografia/Universidade Federal do Paraná, otacilio.paz@ gmail.com

(b) Departamento de Geografia/Universidade Federal do Paraná, tonysampaio@ufpr.br

\section{Eixo: GEOTECNOLOGIAS E MODELAGEM ESPACIAL EM GEOGRAFIA FÍSICA}

\begin{abstract}
Resumo
Os modelos digitais do terreno (MDTs) possuem várias possibilidades de aplicação na Geografia. Destaca-se o seu uso no mapeamento geomorfológico manual ou semiautomático. Mesmo com os constantes avanços científicos e tecnológicos, os MDTs possuem limitações, sendo necessária avaliação de qualidade. Objetiva-se analisar a influência da acurácia altimétrica dos MDTS na geração do Índice de Concentração de Rugosidade Global (ICR Global). Foram utilizados 4 MDTs: SRTM V4, SRTM X, Aster GDEM e um MDT Topogrid gerado por interpolação dos dados planialtimétricos 1:50.000. O MDT Topogrid se enquadrou na escala 1:100.000 e os outros MDTs na escala 1:250.000. Todos os modelos ICRs apresentaram concordância acima de 70\%, com destaque para o ICRTopogrid e ICR-SRTM X que apresentaram concordância acima de $80 \%$. Recomenda-se aplicação do teste em outras áreas de estudo e com o uso de MDTs com diferentes formas de obtenção (tecnologia laser, Drones, RADAR, entre outros).
\end{abstract}

Palavras chave: Geoprocessamento; Geotecnologias; Mapeamento geomorfológico; Relevo.

\section{Introdução}

Os modelos podem ser entendidos como representações simplificadas da realidade, contribuindo para análises dentro da Geografia (CHRISTOFOLETTI, 1999). Os modelos digitais do terreno (MDT) são exemplos desses modelos, representando de forma simplificada a elevação de uma determinada área.

Existem várias diferenças conceituais entre os termos usados para modelos que representem a elevação, sendo as terminologias mais comuns encontrados na literatura: Modelo Digital do Terreno (MDT), Modelo Digital de Elevação (MDE), Digital Elevation Model (DEM) e Modelo Digital de Superfície (MDS) (LI; ZHU; GOLD, 2005; PIKE; EVANS; HENGL, 2009).

Neste trabalho, considera-se o termo MDE como qualquer modelo que armazene dados de elevação sobre uma superfície e o termo MDT utilizado para descrever modelos que tragam dados de elevação em relação a altura média acima do nível do mar ( VALERIANO, 2004; MIRANDA, 2005). 
No entanto, mesmo com avanços científicos e tecnológicos, os MDTs apresentam erros em sua construção, o que trás limitações ou mesmo inviabiliza sua aplicação em determinadas finalidades. Assim, é necessário identificar a grandeza de tais erros bem como informa-la ao usuário. A análise da exatidão altimétrica absoluta é um dos critérios utilizados para avaliação da qualidade dos MDTs (VALERIANO, 2008).

Os MDTs possuem inúmeras possibilidades de aplicação dentro da Geografia, permitindo o mapeamento e análises de paisagens naturais (HENGL; MACMILLAN, 2009), a citar: mapeamento digital do relevo, suscetibilidade à inundação, mapeamento digital de solos, identificação de vulnerabilidade e riscos ambientais, análises de atributos morfométricos do relevo, entre outros (KLINGSEISEN; METTERNICHT; PAULUS, 2008).

Quando ao mapeamento geomorfológico, os MDTs podem servir como apoio a interpretação manual do relevo e até na classificação semiautomáticas a partir de atributos topográficos (declividade, curvatura de vertentes, hipsometria, entre outros) via álgebra de mapas ou de interpoladores (EVANS, 2003; ORTOIMAGENS; PILON, 2006).

A proposta do Índice de Concentração de Rugosidade Global - ICR Global (SAMPAIO; AUGUSTIN, 2014) vem neste sentido, trazendo um método para análise quantitativa do relevo com índice de concordância de até $80 \%$ com a proposta da taxonomia do relevo de Ross (1992) (SILVEIRA et al., 2012). Objetiva-se analisar a qualidade altimétrica de MDTs com distintas formas de obtenção e verificar sua influência na elaboração do ICR Global.

\section{Procedimentos metodológicos}

A área de estudo foi o recorte da folha SG.22-XB-VI, denomina folha Eldorado Paulista, localizada entre os estados do Paraná e São Paulo (figura 1). O recorte de análise está inserido na Província Costeira, abrangendo a baixada litorânea, as serra, os morros isolados e o Vale do Ribeira, constituindo o rebordo do planalto atlântico (ALMEIDA, 1964), com altitudes que variam entre 0m e 1295m. 


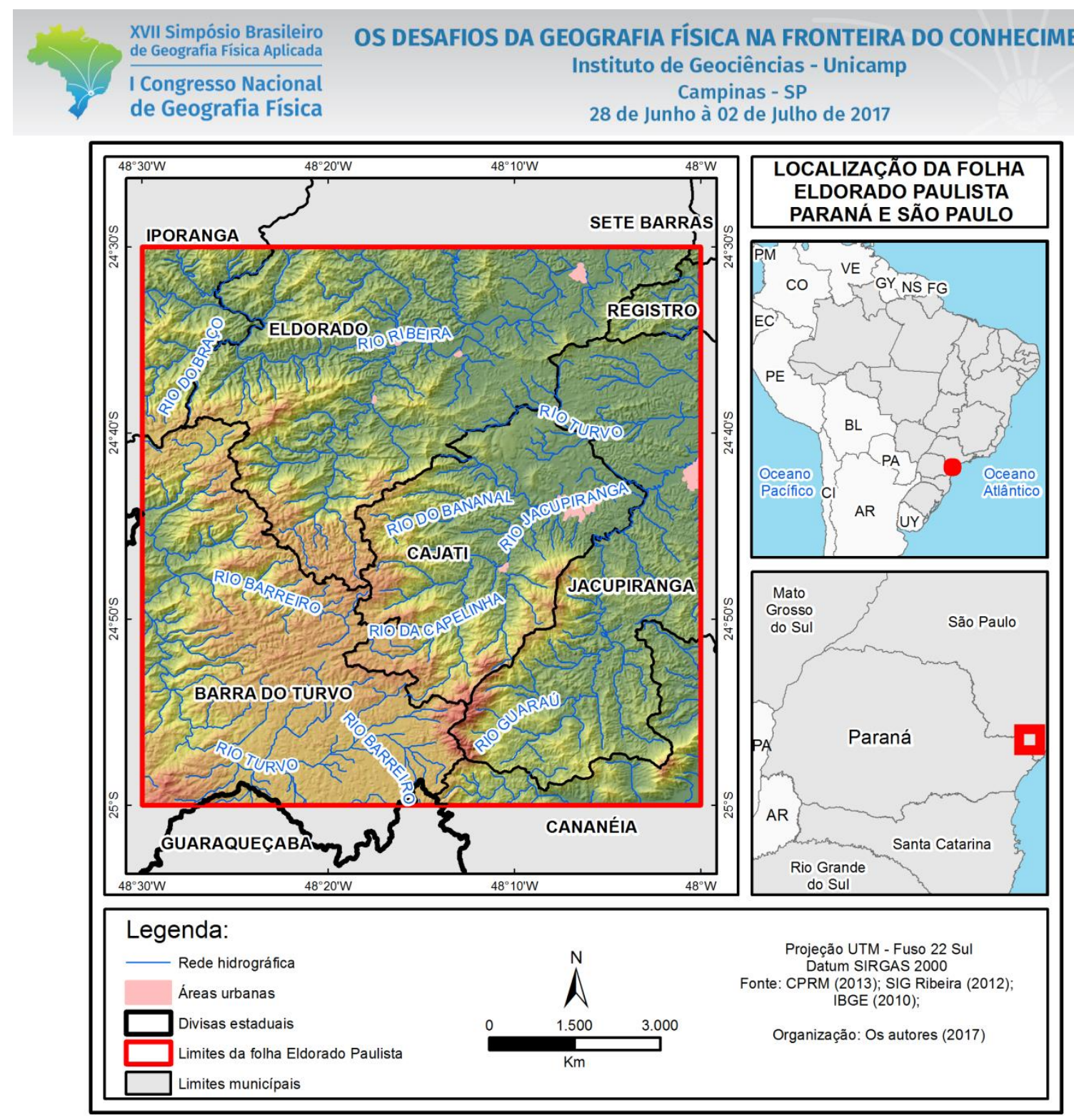

Figura 1 - Localização da área de estudo. Fonte: Os autores (2017)

Para execução das análises espaciais em ambiente de Sistema de Informações Geográficas, foi adotado o software ArcGis versão 10, módulo ArcMap. Foram utilizados 8 MDTs, apresentados no quadro I. Foram analisados 2 modelos do projeto Shuttle Radar Topography Mission (SRTM), diferenciando em sua resolução espacial. O SRTM V4 possui resolução de três segundos de arco, aproximadamente 90 metros. O modelo SRTM X, corresponde aos dados coletados na banda X, correspondendo a um segundo de arco, aproximadamente 30 metros (VALERIANO, 2004).

Tabela I - MDTs utilizados

\begin{tabular}{|c|c|c|}
\hline MDT & Resolução & Fonte \\
\hline SRTM V4 & $90 \mathrm{~m}$ & $\begin{array}{c}\text { Disponibilizado pelo Consortium for Spatial Information } \\
\text { (CGIAR-CSI), dos Estados Unidos da América. }\end{array}$ \\
\hline SRTM X & $30 \mathrm{~m}$ & Disponibilizado pela Deutsches Zentrum für Luft- und \\
\hline
\end{tabular}




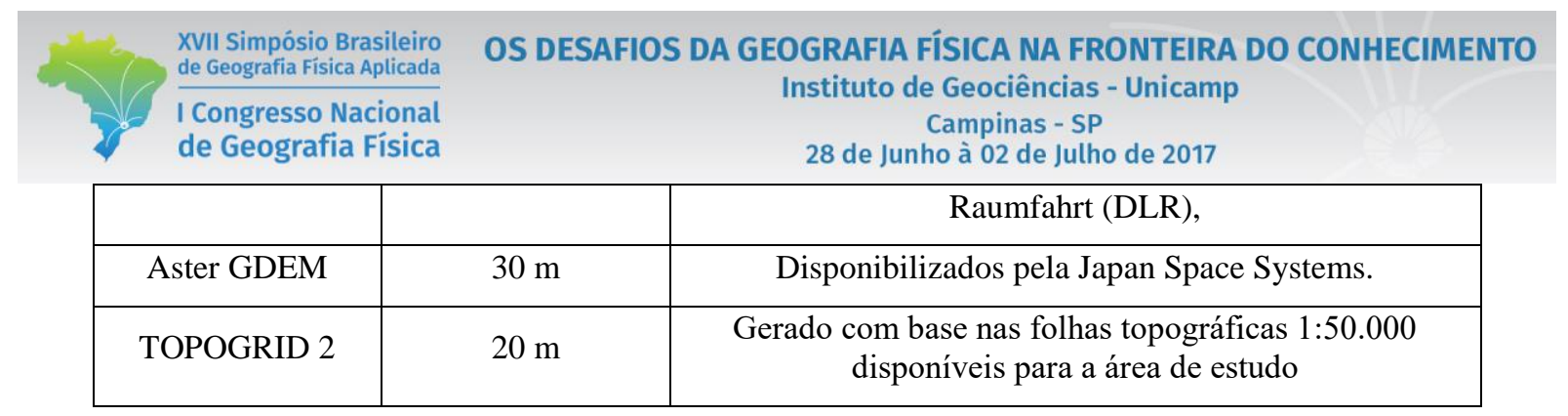

Outro modelo global disponibilizado gratuitamente é o Aster GDEM. Os valores de elevação deste modelo foram obtidos a partir de par estereoscópico de imagens do infravermelho próximo, apresentando resolução horizontal de 30 metros (TACHIKAWA et al., 2011).

O modelo topogrid foi gerado a partir da interpolação de dados planialtimétrico vetoriais (curvas de nível, pontos cotados e hidrografia). As folhas topográficas utilizadas foram: SG-22-X-B-VI-1, SG-22-X-B-VI2, SG-22-X-B-VI-3 e SG-22-X-B-VI-4, na escala 1:50.000 elaboradas pela Diretoria de Serviço Geográfico (DSG). A escolha da resolução horizontal do MDT topogrid foi definida com base nas propostas de HENGL, (2006). As formulas para o calculo da resolução dos MDTs é ilustrada na figura 2.

$$
p=\frac{A}{2 * \sum L}
$$

Onde: $\mathrm{p}$ - tamanho do pixel; $\mathrm{A}$ - área total da área de estudo selecionada; $\sum \mathrm{L}$ - Soma do comprimento de todas as curvas de nível presentes na área de estudo selecionada.

Figura 2 - Formúlas para calcúlo do tamanho do pixel dos MDTs. Fonte: HENGL (2006) e os autores (2017)

A aplicação do método de HENGL (2006) indicou que o pixel ideal para a área de estudo estaria entre 17,5 e 24,2 metros, sendo escolhido o valor de 20 metros. Esse MDT foi gerados a partir da aplicação do algoritmo anudem, baseado na proposta de Huntingford (1988), aplicada no software ArcGis por meio da ferramenta topo to raster.

A avaliação da exatidão altimétrica absoluta utilizou como valores de base as cotas das referencias de níveis (RNs) instalados pelo Instituto Brasileiro de Geografia e Estatística (IBGE). Assim, pode-se aplicar o método proposta pela Especificação Técnica para Estruturação de Dados Geoespaciais Vetoriais (ET ADGV) elaborada pela DSG (DSG, 2016), cuja fórmula é apresentada na figura 3.

$$
p=\frac{A}{2 * \sum L}
$$

Onde: $\mathrm{p}$ - tamanho do pixel; $\mathrm{A}$ - área total da área de estudo selecionada; $\sum \mathrm{L}$ - Soma do comprimento de todas as curvas de nível presentes na área de estudo selecionada.

Figura 3 - Formúlas para calcúlo exatidão altimétrica absoluta. Fonte: (DSG, 2016) 


\section{OS DESAFIOS DA GEOGRAFIA FÍSICA NA FRONTEIRA DO CONHECIMENTO \\ Instituto de Geociências - Unicamp \\ Campinas - SP \\ 28 de Junho à 02 de Julho de 2017}

A partir dos MDTs, foram elaborados os ICRs - Globais seguindo a método proposto por Sampaio e Augustin (2014) (figura 4). A partir dos MDTs, foram gerados modelos de declividade em porcentagem com base nas variáveis direcionais de Horn (1981), sendo esse modelo convertido para a estrutura vetorial numa nuvem de pontos. A nuvem de pontos foi submetida ao estimador de densidade Kernel, passando o resultado pelo processo de normalização. Por fim, o modelo gerado é reclassificado com base na proposta de Sampaio e Augustin (2014), gerando as classes: plano, suave ondulado, ondulado, forte ondulado, escarpado e forte escarpado.

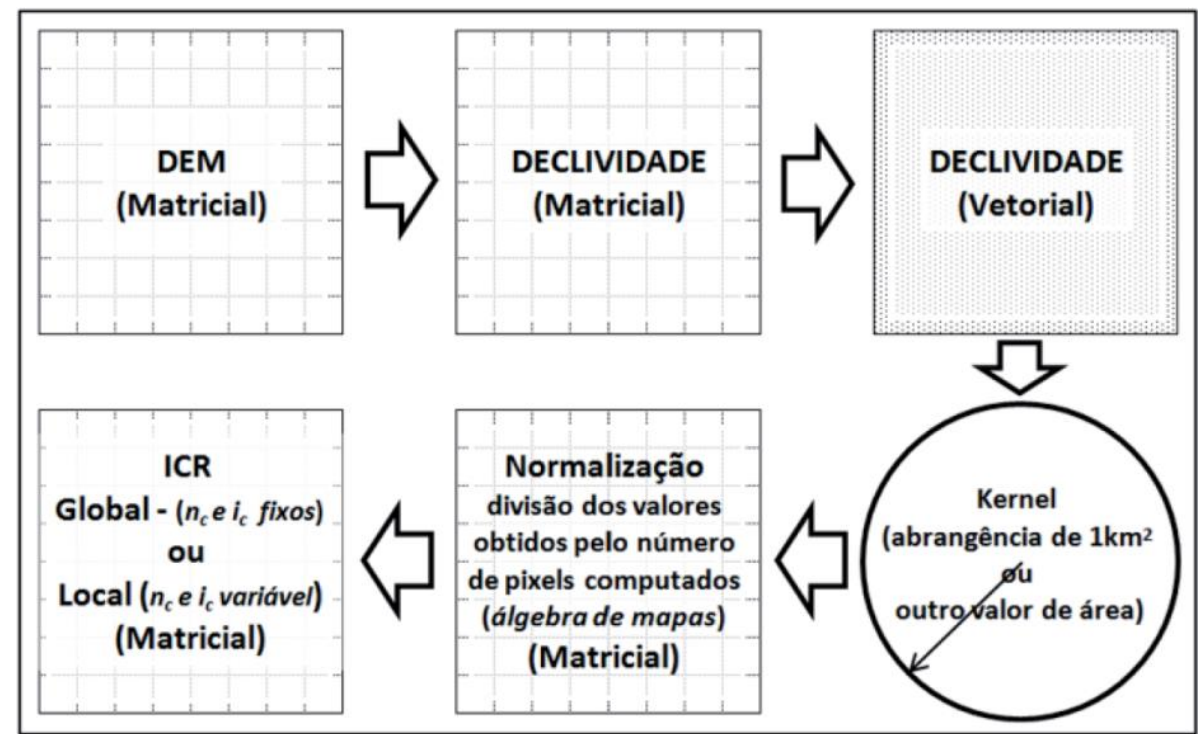

Figura 4 - Formúlas para calcúlo exatidão altimétrica absoluta. Fonte: (SAMPAIO; AUGUSTIN, 2014)

\section{Resultados e discussão}

A exatidão altimétrica absoluta dos MDTs analisados é apresentada nos quadros II e III. De acordo com a norma da ET-ADGV (DSG, 2016), o MDT Topogrid R20 se enquadrou na escala 1:100.000 padrão A, tanto pelo padrão de exatidão cartográfica (PEC) e pela especificação técnica (ET). O MDT que apresentou menor exatidão foi Aster GDEM, se enquadrando na escala 1:250.000 no padrão A (tabela III).

Tabela II - Exatidão altimétrica absoluta dos MDTs analisados

\begin{tabular}{|c|c|c|c|c|}
\hline MDT & $\begin{array}{c}\text { Topogrid R20 } \\
\text { PEC }\end{array}$ & $\begin{array}{c}\text { Topogrid R20 } \\
\text { ET }\end{array}$ & $\begin{array}{c}\text { SRTM X } \\
\text { PEC }\end{array}$ & $\begin{array}{c}\text { SRTM X } \\
\text { ET }\end{array}$ \\
\hline Valor & 11,4658 & 7,0159 & 16,2587 & 13,1062 \\
\hline Padrão "A" & $1: 100.000$ & $1: 100.000$ & $1: 250.000$ & $1: 250.000$ \\
\hline Padrão "B" & - & - & $1: 100.000$ & $1: 100.000$ \\
\hline Padrão "C" & $1: 50.000$ & $1: 50.000$ & - & - \\
\hline
\end{tabular}


Tabela III - Exatidão altimétrica absoluta dos MDTs analisados

\begin{tabular}{|c|c|c|c|c|}
\hline MDT & $\begin{array}{c}\text { SRTM V4 } \\
\text { PEC }\end{array}$ & $\begin{array}{c}\text { SRTM V4 } \\
\text { ET }\end{array}$ & $\begin{array}{c}\text { Aster GDEM } \\
\text { PEC }\end{array}$ & $\begin{array}{c}\text { Aster GDEM } \\
\text { ET }\end{array}$ \\
\hline Valor & 20,0255 & 15,2598 & 24,2584 & 15,8681 \\
\hline Padrão "A" & $1: 250.000$ & $1: 250.000$ & $1: 250.000$ & $1: 250.000$ \\
\hline Padrão "B" & - & $1: 100.000$ & - & $1: 100.000$ \\
\hline Padrão "C" & $1: 100.000$ & - & - & - \\
\hline Padrão "D" & 1 & - & $1: 100.000$ & - \\
\hline
\end{tabular}

Os modelso ICRs elaborados são apresentados na figura 5. De modo geral, a área de estudo apresenta relevo mais escarpado na porção noroeste, leste e central, e as áreas onduladas nas porções sul-sudoeste e nordeste. A exatidão altimétrica absoluta dos MDTs analisados é apresentada na tabela II. 


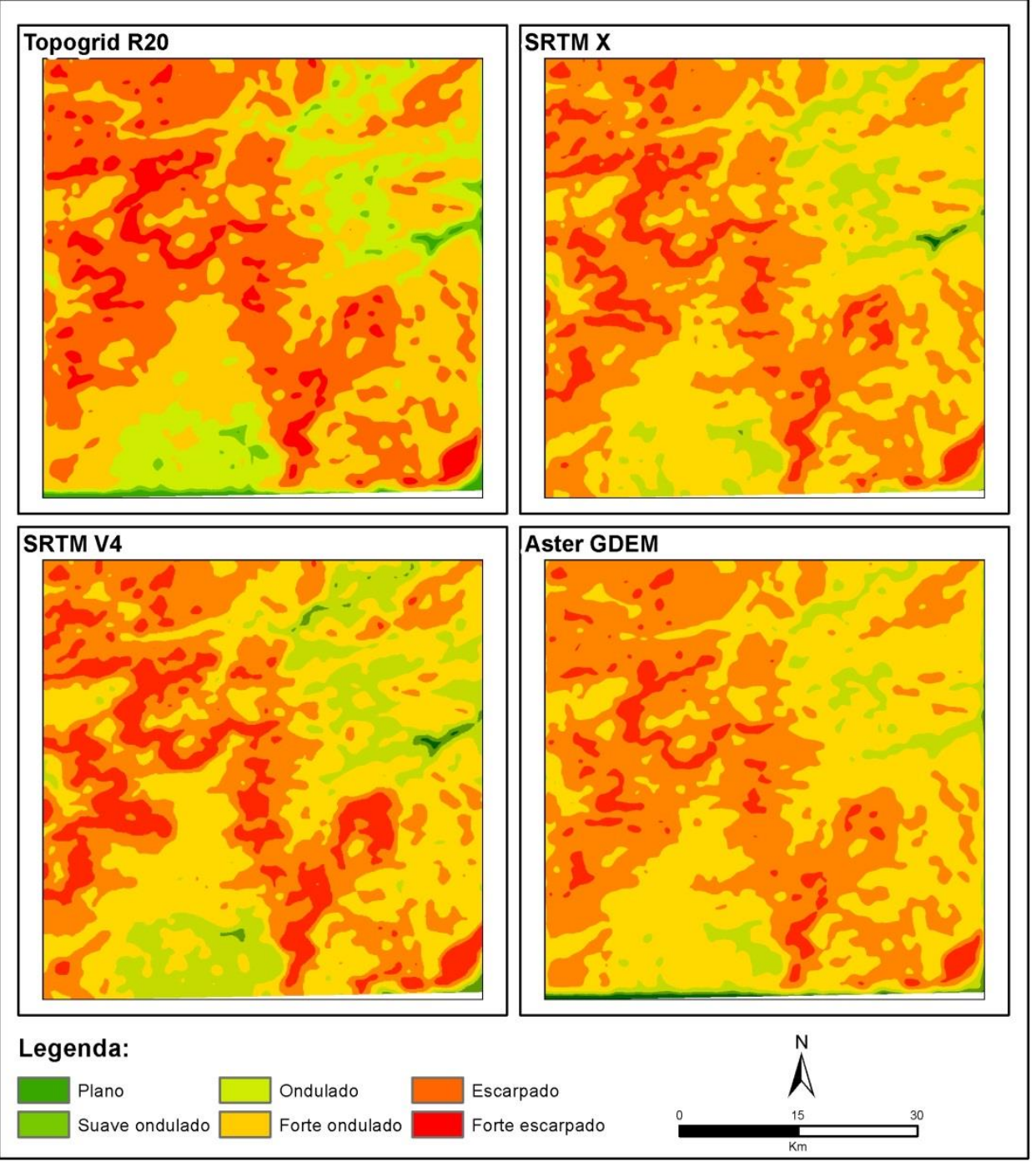

Figuras 5 - ICRs - Globais gerados para a área de estudo. Fonte: Os autores (2017).

Cabe destacar que o MDT SRTM X se enquadrou na mesma escala que o MDT SRTM V4 (1:250.000), ou seja, a melhora na resolução horizontal do MDT SRTM X não interferiu no resultado da análise do padrão de exatidão altimética.

O ganho de qualidade altimétrica ocorreu com a elaboração do MDT topogrid R20, se enquadrando na escala 1:100.000, apresentando melhores resultados que os MDTs SRTM V4, SRTM X e Aster GDEM. No entanto, cabe ressaltar que os dados originais usados na interpolaçao estavam na escala 1:50.000, ou seja, mesmo apresentando melhores resultados, esse MDT perdeu qualidade no processo de interpolação, se enquadrando na escala 1:50.000 apenas no Padrão C. 
XVII Simpósio Brasileiro de Geografia Fisica Aplicada

I Congresso Nacional de Geografia Física
OS DESAFIOS DA GEOGRAFIA FÍSICA NA FRONTEIRA DO CONHECIMENTO

Instituto de Geociências - Unicamp

Campinas - SP

28 de Junho à 02 de Julho de 2017

A figura 6 ilustra as paisagens representadas pelo ICR. Os pontos 1, 2 e 3 apresentam áreas classificadas como "Ondulada". O ponto 4 representa as áreas classificadas como "Plano", na porção oeste da área de estudo. O ponto apresenta as áreas classificadas como "Escarpado e Forte escarpado".

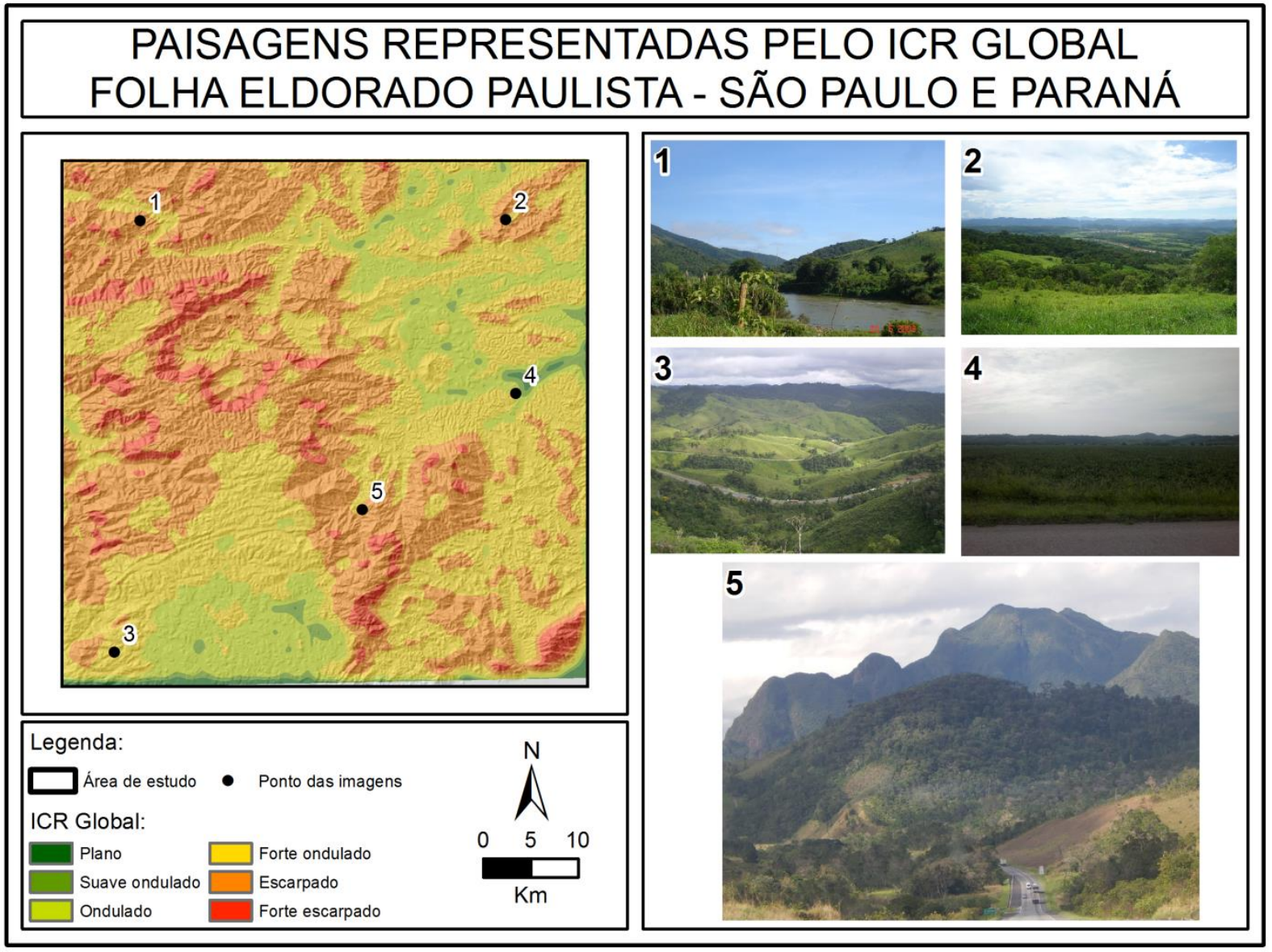

Figuras 6 - ICRs - Globais gerados para a área de estudo. Fonte: Os autores (2017); Imagens Google Panoramico $(\mathrm{s} / \mathrm{n})$.

O quadro IV apresenta as áreas de cada classe do ICR de acordo com o MDT empregado. De forma geral predominam as áreas classificas como "Ondulado e Forte ondulado". Em contraposição, as áreas classificadas como "Plano" são pouco representativas na área de estudo.

Tabela IV - Exatidão altimétrica absoluta dos MDTs analisados

\begin{tabular}{|c|c|c|c|c|c|c|}
\hline ICR & Plano & $\begin{array}{c}\text { Suave } \\
\text { ondulado }\end{array}$ & Ondulado & $\begin{array}{c}\text { Forte } \\
\text { ondulado }\end{array}$ & Escarpado & $\begin{array}{c}\text { Forte } \\
\text { escarpado }\end{array}$ \\
\hline SRTM V4 & 1,6 & 25,1 & 372,2 & 1117,2 & 914,2 & 376,3 \\
\hline SRTM X & 21,8 & 46,0 & 231,1 & 1250,5 & 1118,7 & 138,5 \\
\hline Aster GDEM & 19,5 & 37,7 & 133,8 & 1239,2 & 1230,1 & 146,2 \\
\hline
\end{tabular}




$\begin{aligned} & \text { XVII Simpósio Brasileiro } \\
& \text { de Geografia Fisica Aplicada }\end{aligned}$
$\begin{aligned} & \text { I Congresso Nacional } \\
& \text { de Geografia Física }\end{aligned}$
\begin{tabular}{|l|c|c|c|c|c|c|}
\hline TOPOGRID R20 & 31,1 & 62,1 & 328,0 & 1006,6 & 1117,9 & 260,8 \\
\hline
\end{tabular}

A figura 7 ilustra a áreas concordantes e divergentes entre os modelos ICR gerados. Os ICRs gerados a partir dos MDTs SRTM X e Aster Gdem apresentaram maior concordância, cerca de 87\%. Os ICRs gerados pelos MDTs SRTM V4 (SRTM 90) e Aster Gdem apresentam menores valores de concordância, cerca de $73 \%$.

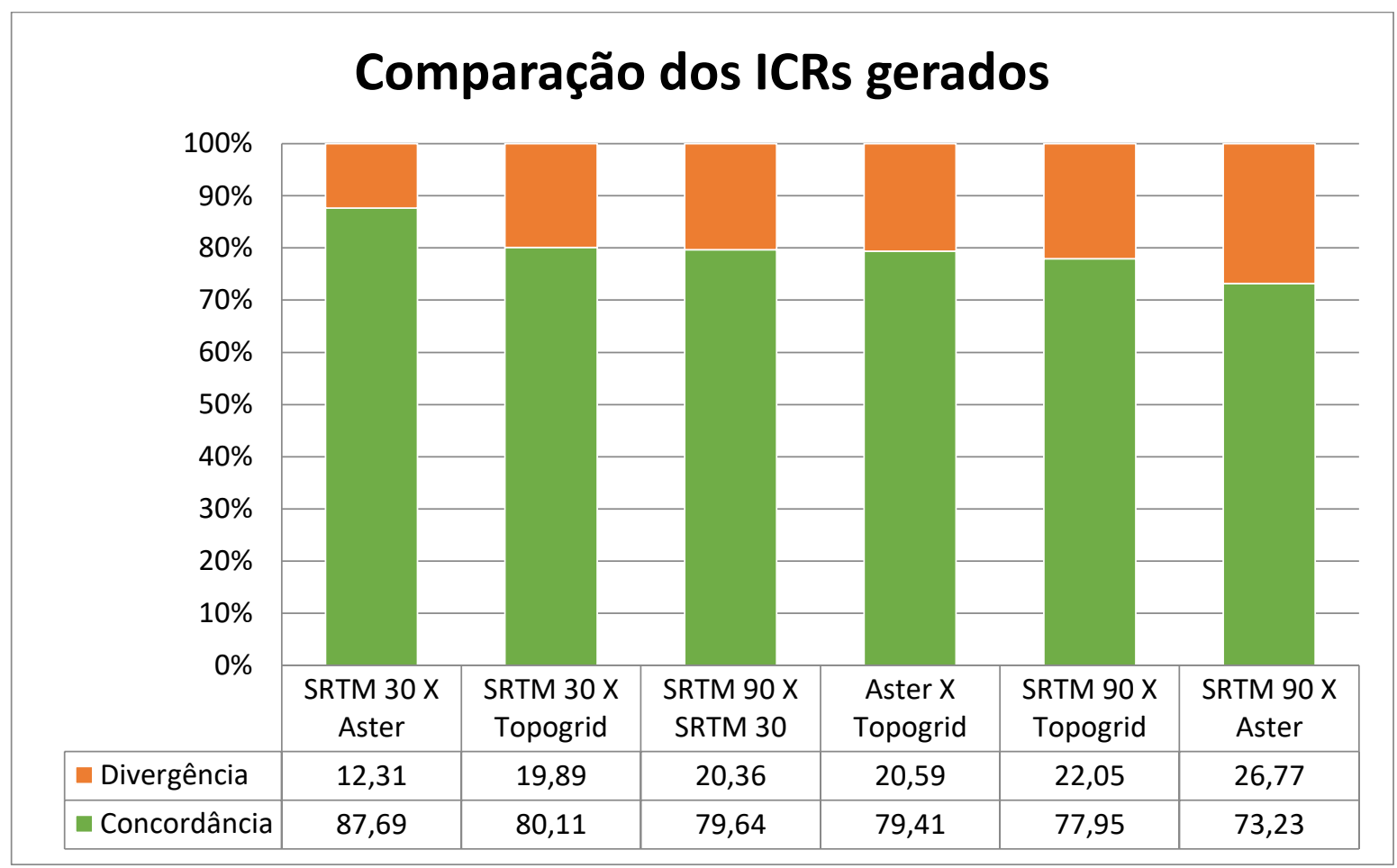

Figuras 7 - ICRs - Globais gerados para a área de estudo. Fonte: Os autores (2017).

O modelo ICR gerado a partir do MDT SRTM X (Escala 1:250.000) e o modelo ICR gerado a partir do MDT topogrid R20 (escala 1:100.000), apresentaram valores de área entre as classes do relevo semelhantes, comprovando pela concordância de classificação de aproximadamente $80 \%$. Ou seja, o modelo ICR gerado a partir de um MDT interpolado de uma escala 1:50.000 teve classificação semelhante ao modelo ICR gerado a partir de MDTs globais.

\section{Considerações finais}

Os modelos de ICRs Globais gerados para a área de estudo apresentaram grande semelhança, superior a $70 \%$ de concordância em todos os casos. Destaque para a aplicação do modelo ICR Global na quantificação e classificação do relevo. 
Mesmo se enquadrando na escala 1:100.000, o modelo ICR Global elaborado a partir do MDT topogrid R20 apresentou valores de concordância elevados de aproximadamente entre $70 \%$ e $80 \%$ em relação aos modelos ICR Global gerados a partir de MDTs globais tais como o SRTM V4, SRTM X e Aster GDEM.

Recomenda-se a repetição da análise entre MDTs interpolados e MDTs globais, aplicados em outras áreas de estudo com configurações de relevo distintas e em outras escalas, a fim de se investigar sua influência na elaboração o ICR Global.

\section{Bibliografia}

ALMEIDA, F. F. M. Fundamentos geológicos do relevo paulista. Boletim do Instituto Geográfico Geológico, v. 41, p. 169-263, 1964.

CHRISTOFOLETTI, A. Modelagem de sistemas ambientais. 1. ed. [s.1.] Edgard Blücher, 1999.

DSG - DIRETORIA DO SERVIÇO GEOGRÁFICO. Especificação técnica para a aquisição de dados geoespaciais vetoriais (ET-ADGV). Ministério da Defesa, Exército Brasileiro, Departamento de Ciência e Tecnologia. Brasília-DF, 2a edição, v. 2, 2016.

EVANS, I. S. Scale-specific landforms and aspects of the land surface. Concepts and Modelling in Geomorphology: International Perspectives. Terrapub, Tokyo, p. 61-84, 2003.

HENGL, T. Finding the right pixel size. Computers and Geosciences, v. 32, n. 9, p. 1283-1298, 2006.

HENGL, T.; MACMILLAN, R. A. Geomorphometry-a key to landscape mapping and modelling. [s.l: s.n.]. v. 33

HORN, B. K. P. Hill shading and the reflectance map. Proceedings of the IEEE, v. 69, n. 1, p. 14-47, 1981.

HUNTINGFORD, C. Calculation of hydrologically sound digital elevation models. Proceedings of the Third International Symposium on ..., p. 117-133, 1988.

KLINGSEISEN, B.; METTERNICHT, G.; PAULUS, G. Geomorphometric landscape analysis using a semiautomated GIS-approach. Environmental Modelling and Software, v. 23, n. 1, p. 109-121, 2008.

LI, Z.; ZHU, Q.; GOLD, C. M. Digital terrain modelling. Principles and methodology. [s.l: s.n.].

MIRANDA, E. E. DE. Brasil em relevo. Campinas: Embrapa Monitoramento por Satélite, 2005.

ORTO-IMAGENS, G. D. E.; PILON, J. A. GERAÇÃO DE MODELOS NUMÉRICOS DE ELEVAÇÕES. Poligrafo Aerofotogrametria, v. 8, p. 1-10, 2006.

PIKE, R. J.; EVANS, I. S.; HENGL, T. Geomorphometry: A brief guide. [s.l: s.n.]. v. 33

ROSS, J. A. S. O registro cartográfico e a questão da taxonomia do relevo. Revista de Geografia, São, 1992. 


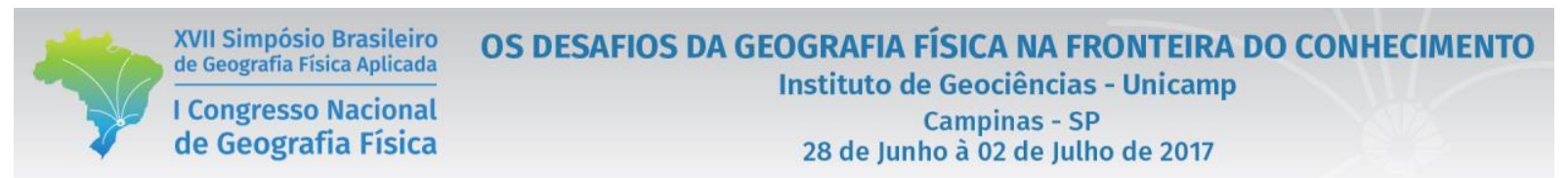

SAMPAIO, T. V. M.; AUGUSTIN, C. H. R. R. Índice de concentração da rugosidade: uma nova proposta metodológica para o mapeamento e quantificação da dissecação do relevo como subsídio a cartografia geomorfologica. Revista Brasileira de Geomorfologia, v. 15, n. 1, p. 1-14, 2014.

SILVEIRA, R. M. P. et al. ANÁliSE DIGITAL DO RELEVO EMPREGADA NO MAPEAMENTO DE UNIDADES GEOMORFOLÓGICAS. Geografar, v. 7, p. 43-68, 2012.

TACHIKAWA, T. et al. Characteristics of ASTER GDEM version 2International Geoscience and Remote Sensing Symposium (IGARSS). Anais...2011

VALERIANO, M. Modelo digital de elevação com dados srtm disponíveis para a américa do sul. 15016334249, p. 72, 2004.

VALERIANO, M. DE M. Dados topográficos. Geomorfologia: conceitos e tecnologias atuais. São Paulo: Oficina de Textos, p. 72-104, 2008. 